\title{
Neuroprotective effect of Angelica gigas root in a mouse model of ischemic brain injury through MAPK signaling pathway regulation
}

\author{
Se-Eun Lee ${ }^{1 \dagger}$, Jung-Hoon Kim ${ }^{1 \dagger}$, Chiyeon Lim² and Suin Cho ${ }^{1 *}$ (D)
}

\begin{abstract}
Background: The root of Angelica gigas Nakai (Apiaceae) has been traditionally used as an important herbal medicine to treat blood-deficiency-related disorders in Eastern Asian countries, and recently, it has been recognized as a potential candidate for improving cardiovascular diseases.

Methods: In this study, the neuroprotective effect of a methanol extract of A. gigas root (RAGE) was investigated in a mouse stroke model induced by a 90 min transient middle cerebral artery occlusion (tMCAO). Infarction volumes and morphological changes in brain tissues were measured using TTC, cresyl violet, and H\&E staining. The neuroprotective mechanism of RAGE was elucidated through investigation of protein expression levels using western blotting, $I H C$, and ELISA assays. The plasma concentrations of decursin, a major compound in RAGE, were measured after oral administration of RAGE to SD rats.
\end{abstract}

Results: The infarction volumes in brain tissues were significantly reduced and the morphological deteriorations in the brain neuron cells were improved in tMCAO mice when pre-treated with RAGE at $1000 \mathrm{mg} /(\mathrm{kg} \mathrm{bw} \cdot \mathrm{d}$ ) for two consecutive days. The neuroprotective mechanism of RAGE was confirmed to attenuate ERK-related MAPK signaling pathways in the ipsilateral hippocampus hemisphere in mice. The concentrations of decursin in rat plasma samples showed peak absorption and elimination in vivo after oral administration of RAGE at $100 \mathrm{mg} / \mathrm{rat}$.

Conclusion: Mice administered RAGE before the TMCAO operation had less neuronal cell death than those that were not administered RAGE prior to the operation, and this study provides preclinical evidence for use of $A$. gigas in ischemic stroke.

Keywords: Angelica gigas (apiaceae), Ischemic stroke, Infarction, Neuroprotection, Pharmacokinetics

\section{Background}

Acute ischemic cerebral stroke is considered the leading cause of morbidity and mortality in modern society [1-5]. When ischemic stroke occurs, cerebral inflammation and cell death are induced in the ischemic

\footnotetext{
*Correspondence: sicho@pusan.ac.kr

† Se-Eun Lee and Jung-Hoon Kim contributed equally to this study

${ }^{1}$ Department of Korean Medicine, School of Korean Medicine, Yangsan Campus of Pusan National University, Yangsan 50612, Republic of Korea

Full list of author information is available at the end of the article
}

lesions, and inflammatory signals are activated by harmful stimuli such as arterial occlusion [6-9].

Ischemic stroke causes high morbidity and disability, with many patients needing rehabilitation and longterm care resulting in a massive financial burden on healthcare systems $[9,10]$. Thrombolytic agents, such as anticoagulant or antiplatelet agents, have been used for the treatment of stroke with some success. However, there are limitations to their use, including side effects such as hemorrhage, and the limited window of opportunity for efficacious administration. Thus, further

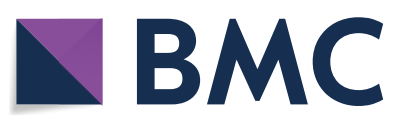

(c) The Author(s) 2020. This article is licensed under a Creative Commons Attribution 4.0 International License, which permits use, sharing, adaptation, distribution and reproduction in any medium or format, as long as you give appropriate credit to the original author(s) and the source, provide a link to the Creative Commons licence, and indicate if changes were made. The images or other third party material in this article are included in the article's Creative Commons licence, unless indicated otherwise in a credit line to the material. If material is not included in the article's Creative Commons licence and your intended use is not permitted by statutory regulation or exceeds the permitted use, you will need to obtain permission directly from the copyright holder. To view a copy of this licence, visit http://creativecommons.org/licenses/by/4.0/. The Creative Commons Public Domain Dedication waiver (http://creativecommons.org/publicdomain/zero/1.0/) applies to the data made available in this article, unless otherwise stated in a credit line to the data. 
research on the prevention and treatment of ischemic stroke is essential $[11,12]$.

Angelica gigas Nakai (Apiaceae), also called Korean angelica, is a perennial plant from Asian countries, and in traditional herbal medicine, its roots are used to treat symptoms or disorders caused by blood-deficiency [13]. Several studies have investigated the chemical components and pharmacological activities of $A$. gigas [13-21]. In Korea, the root of $A$. gigas (RAG) has traditionally been used as a representative blood circulation agent when derived from either source [13, 14].

According to traditional Korean medicine, strokes are mainly caused by blood stasis, and RAG has been widely used by traditional Korean medicine practitioners to treat ischemia-related diseases [13-15]. Pharmacologically, it has been reported to have anticancer and antibacterial effects, antioxidant activity, and to improve circulatory diseases activity [15-19]. More recently, research has indicated that its components have neuroprotective and anti-inflammatory effects [20,21].

Therefore, in this study, we investigated the mechanism of neuroprotective action of the methanolic extract of the root of $A$. gigas (RAGE) on a mouse model of ischemic stroke induced by a $90 \mathrm{~min}$ transient middle cerebral artery occlusion (tMCAO). Moreover, pharmacokinetic parameters of decursin, a dominant compound in $A$. gigas root, were determined by comparing plasma samples from rats orally administered RAGE at a dose corresponding to that administered to $\mathrm{TMCAO}$ mice.

\section{Materials and methods}

\section{Chemicals and reagents}

Analytical methanol, acetonitrile, and water were purchased from J.T. Baker Inc. (Phillipsburg, NJ, USA). LC/ MS grade acetonitrile and water (containing $0.1 \%$ formic acid) were purchased from Fisher Scientific (Pittsburgh, PA, USA). Ethyl acetate was obtained from SK Chemicals (Seongnam, Gyeonggi-do, Korea). Trifluoroacetic acid was purchased from Sigma-Aldrich (St Louis, MO, USA). Nodakenin, decursin, and schizandrin (internal standards, IS) were purchased from the Korea Food and Drug Administration (Osong, Chungbuk, Korea). Chlorogenic acid and imperatorin were purchased from ChemFace (Wuhan, Hubei, China). All marker compounds had $\geq 98 \%$ purity.

PBS was purchased from Bio Basic Inc. (Markham, Ontario, Canada). 2,3,5-Triphenyl-tetrazolium chloride (TTC) and cresyl violet were purchased from SigmaAldrich (St. Louis, MO, USA). Saline was obtained from JW Pharmaceutical Co., Ltd. (Seoul, Korea). The optimal cutting temperature compound for cryostat embedding medium was purchased from Thermo Fisher Scientific (Waltham, MA, USA). Methanol was purchased from
SK chemicals (Ulsan, Korea). Protein extraction solution was purchased from iNtRON (Seongnam-si, Gyeonggido, Korea). Primary antibodies for $p$-ERK (\#9101), ERK (\#9102), $p$-JNK (\#9255), JNK (\#9252), p-p38 (\#9211), and p38 (\#9212) were purchased from Cell Signaling Technology (Danvers, MA, USA). Goat polyclonal secondary anti-rabbit (ADI-SAB-301-J) and anti-mouse (ADI-SAB101-J) IgG antibodies were obtained from Enzo Life Sciences Inc. (Farmingdale, NY, USA). West-Q chemiluminescent substrate was purchased from GenDEPOT (Katy, TX, USA). BCA reagent, BSA standard, and enhanced chemiluminescence western blotting chemiluminescent substrate were purchased from Thermo Fisher Scientific (Waltham, MA, USA). Enzyme-linked immunosorbent assay (ELISA) kits for TNF- $\alpha$ and IL- 6 was purchased from Abcam (Cambridge, UK).

\section{Preparation of RAGE}

The dried root of $A$. gigas was purchased from the Kwangmyungdang Medical Herbs Co. (Namgu, Ulsan, Korea). RAG (200 g) was extracted with $99 \%$ methanol at $25^{\circ} \mathrm{C}$ for two days to obtain the methanolic extract. The extract was filtered and concentrated again using a rotary vacuum evaporator (EYELA, Tokyo, Japan), and then, the concentrate obtained ( $34 \mathrm{~g}$ ) was designated RAGE.

\section{Preparation of RAGE for high performance liquid chromatography (HPLC) analysis and analytical conditions}

RAGE (10 mg) was dissolved in methanol $(1 \mathrm{~mL})$ and filtered through $0.2 \mu \mathrm{m}$ syringe filters (BioFact, Daejeon, Korea). The filtrate was prepared for HPLC analysis at a final concentration of $1000 \mu \mathrm{g} / \mathrm{mL}$ by dilution with methanol.

HPLC analysis of four marker compounds was performed using an Agilent 1200 liquid chromatography system (Agilent Technologies, Palo Alto, CA, USA) equipped with an autosampler, degasser, quaternary solvent pump, and diode array detector. Separation of the marker compounds was performed on a Capcell Pak $\mathrm{Mg}$ II $_{18}$ column ( $4.6 \mathrm{~mm} \times 250 \mathrm{~mm}, 5 \mu \mathrm{m}$; Shiseido, Tokyo, Japan) at $35^{\circ} \mathrm{C}$ using a flow rate and an injection volume set at $1 \mathrm{~mL} / \mathrm{min}$ and $10 \mu \mathrm{L}$, respectively. The mobile phase consisted of water containing $0.1 \%$ trifluoroacetic acid (solvent $\mathrm{A}$ ) and acetonitrile (solvent $\mathrm{B}$ ) and the gradient elution was as follows: $10 \%$ (B) for 0-5 $\mathrm{min}, 10-20 \%$ (B) for $5-10 \mathrm{~min}, 20 \%$ (B) for $10-33 \mathrm{~min}, 20-50 \%$ (B) for $33-35 \mathrm{~min}, 50 \%$ (B) for $35-60 \mathrm{~min}, 50-90 \%$ (B) for 60-61 $\mathrm{min}, 90 \%$ (B) for 61-65 min, and then, re-equilibrated to $10 \%$ (B) until $70 \mathrm{~min}$. The diode-array detector was set at ultraviolet wavelengths of $250 \mathrm{~nm}$ (for imperatorin), $325 \mathrm{~nm}$ (for chlorogenic acid), $330 \mathrm{~nm}$ (for decursin), and $335 \mathrm{~nm}$ (for nodakenin). 


\section{Experimental animals}

Wild-type male C57BL/6 mice and SD rats were supplied by Samtako (Incheon, Korea). Animals were housed at constant temperature $\left(22 \pm 2{ }^{\circ} \mathrm{C}\right)$ and relative humidity $(50 \pm 10 \%)$ conditions under a $12 \mathrm{~h}$ light/dark cycle for 7 days with free access to feed and water before the experiments. Animals were fasted overnight before surgery or oral administration of RAGE. Animal experiments were conducted according to the guidelines for animal experimentation issued by the Pusan National University and the experimental procedures were approved beforehand by the Animal Ethics Committee of Pusan National University (PNU-2018-2113 and PNU-2019-2124).

\section{Induction of tMCAO}

Mice (aged 7 to 9 weeks, weighing 21 to $25 \mathrm{~g}$ ) were exposed to $90 \mathrm{~min}$ tMCAO with an intraluminal filament. Mice were anesthetized using 1.5-2\% isoflurane in $\mathrm{N}_{2} \mathrm{O} / \mathrm{O}_{2}$ (70\%/30\%). In anesthetized mice, the left side of the common carotid artery was exposed and isolated. The middle cerebral artery (MCA) was occluded by inserting an 8-0 surgical coated-monofilament nylon suture into the internal carotid artery, which was advanced further until it closed the origin of the MCA. During the experiments, MCA blood flow was monitored using a laser Doppler flowmeter (MoorVMS-LDF, Moor Instruments Ltd., UK) connected to a single fiber optic probe adhered onto the skull surface of the core area supplied by the left. The reduction and maintenance of relative cerebral blood flow (rCBF) under $20 \%$ of pre-ischemic baseline was used to confirm the occlusion, and $\mathrm{rCBF}$ recovery more than $40 \%$ of the pre-ischemic baseline was considered as successful reperfusion. Rectal temperature was maintained at $37 \pm 0.5^{\circ} \mathrm{C}$ by means of a heating blanket and heating lamp throughout the surgery. Sham-operated control mice received the same surgical procedure without insertion of a filament.

\section{TTC staining}

Mice were quickly anesthetized with isoflurane, and the brains were removed rapidly and placed in ice-cold water for $3 \mathrm{~min}$. Coronal slices of $1 \mathrm{~mm}$ thickness were prepared, and sections were immersed in $2 \%$ TTC at $37^{\circ} \mathrm{C}$ for $17 \mathrm{~min}$. The presence of infarction was determined by the area that was stained negative with TTC.

\section{Neurological deficit scores (NDS) assessment}

NDS assessments were performed by investigators who were blinded to the experimental groups, as described previously [22]. The following rating scale was used: $0=$ no deficit, $1=$ failure to extend right forepaw,
$2=$ decreased grip strength of right forepaw, $3=$ circling to right by pulling the tail, and $4=$ spontaneous circling.

\section{Western blot analysis}

The ipsilateral cortex and hippocampus of tMCAO mice were homogenized in PRO-PREP protein extraction solution. After centrifugation, the supernatants were used for immunoblot analysis. $p$-ERK, $p$-JNK, JNK, $p$ p38, p38 (diluted 1:2000), and ERK (diluted 1:1000) were used as the primary antibodies. Immunodetection was performed and quantified using a photosensitive luminescent analyzer system (Amersham ${ }^{\mathrm{TM}}$ Imager 600, MA, USA) according to the protocol provided by the manufacturer. All bands were analyzed using Image $(\mathrm{NIH}$, MD, USA).

\section{Cresyl violet, hematoxylin and eosin (H\&E), and immunohistochemistry $(\mathrm{IHC})$ staining}

For cresyl violet staining, sections were incubated at $40^{\circ} \mathrm{C}$ for $10 \mathrm{~min}$. Slides were placed directly in chloroform:alcohol overnight. Then, the sections were stained with cresyl violet at $40^{\circ} \mathrm{C}$ for $40 \mathrm{~min}$. After washing under water for $10 \mathrm{~s}$, sections were quickly dehydrated in a 95\% alcohol series, and placed in xylene for $1 \mathrm{~min}$. The slides were sealed with a cover slip and mounting solution. Differences between the treatment groups were compared under an optical microscope.

For $\mathrm{H} \& \mathrm{E}$ staining, sections were incubated at $40^{\circ} \mathrm{C}$ for $10 \mathrm{~min}$. Then, slides were passed through $85 \%$ alcohol for rehydration, and washed under water for $2 \mathrm{~min}$. The sections were stained with hematoxylin for $10 \mathrm{~min}$ and then washed under water for $1 \mathrm{~min}$ to remove the excess dye from the tissue. The sections were stained with eosin Y solution for $1 \mathrm{~min}$. After washing under running water for $1 \mathrm{~min}$, air-dried sections were passed through $85 \%$ and $99 \%$ alcohol series and dewaxed in xylene for $1 \mathrm{~min}$. The slides were sealed with a cover slip and mounting solution. Differences between the treatment groups were compared under an optical microscope.

For IHC staining, primary antibodies for TNF- $\alpha$ and IL-6 were used at a 1:200 dilution. The sections were incubated overnight at $-4{ }^{\circ} \mathrm{C}$ in primary antibodies. The sections were washed, and incubated with secondary antibody for $1 \mathrm{~h}$ each at room temperature. IHC analysis was performed using the DAKO Envision kit (DAKO, Carpinteria, CA, USA).

\section{ELISA assay}

To detect TNF- $\alpha$ or IL-6 in ipsilateral hippocampus proteins and blood serum, we used an ELISA kit. The 
samples and standard samples were diluted with distilled water and applied to ELISA plates. The TNF- $\alpha$ or IL- 6 concentrations were determined according to the manufacturer's instructions. Absorbance levels were measured at $450 \mathrm{~nm}$ using an ELISA reader.

\section{LC/MS conditions for the pharmacokinetics study}

Quantification of decursin was performed using an Accela LC system (Thermo Fisher Scientific; MA, USA) equipped with an autosampler, degasser, and quaternary solvent pump. Decursin and IS were separated on a Hypersil GOLD $\mathrm{C}_{18}$ column $(2.1 \mathrm{~mm} \times 100 \mathrm{~mm}, 1.9 \mu \mathrm{m}$; Thermo Fisher Scientific, MA, USA) at $35^{\circ} \mathrm{C}$. The flow rate was set at $300 \mu \mathrm{L} / \mathrm{min}$ with an injection volume of 5 $\mu \mathrm{L}$. The mobile phase consisted of water (containing $0.1 \%$ formic acid, A) and acetonitrile (B) and the gradient elution applied was as follows: $30-60 \%$ (B) for $0-4 \mathrm{~min}, 60 \%$ (B) for $0.5 \mathrm{~min}$, and then re-equilibrated to $30 \%$ (B) until the end of the analysis.

An LCQ Fleet ion-trap mass spectrometer (Thermo Fisher Scientific; MA, USA) was used to detect compounds in the eluent using the electrospray ionization source in the positive-ion mode. MS conditions were as follows: sheath gas (nitrogen), 50 arbitrary units; auxiliary gas (nitrogen), 20 arbitrary units; spray voltage, $5.0 \mathrm{kV}$; capillary temperature, $300^{\circ} \mathrm{C}$; and capillary voltage, $30.0 \mathrm{~V}$. Quantification of the compound was performed in the selective ion monitoring mode at $329 \mathrm{~m} / z$ $[\mathrm{M}+\mathrm{H}]^{+}$for decursin and $415 \mathrm{~m} / z[\mathrm{M}+\mathrm{H}]^{+}$for the IS. Data was processed using Excalibur (v. 2.1.0; Thermo Fisher Scientific, CA, USA).

\section{Preparation of stock solutions, calibration standards, and plasma sample}

Accurately weighed decursin and IS were dissolved in methanol at a concentration of $100 \mu \mathrm{g} / \mathrm{mL}$, and the solution was diluted with methanol to prepare working solutions for the construction of the calibration curve. Calibration standards were prepared using $150 \mu \mathrm{L}$ of blank rat plasma spiked with $10 \mu \mathrm{L}$ of working solutions and $10 \mu \mathrm{L}$ of IS solution, which was extracted using ethyl acetate.

Plasma samples were prepared using a liquid-liquid extraction method. An aliquot of $150 \mu \mathrm{L}$ plasma sample was spiked with $10 \mu \mathrm{L}$ of IS solution and $10 \mu \mathrm{L}$ of methanol, and $800 \mu \mathrm{L}$ of ethyl acetate was added. Extraction of decursin and IS was performed by vortex mixing for $2 \mathrm{~min}$ at $25^{\circ} \mathrm{C}$, followed by centrifugation at $704 \times g$ for $10 \mathrm{~min}$ at $20^{\circ} \mathrm{C}$. The upper layer (ethyl acetate) was then transferred to a clean $1.5 \mathrm{~mL}$ polypropylene tube and evaporated using a nitrogen gas blowing concentrator (MGS-2200; EYELA, Miyagi, Japan). The residue obtained after evaporation was reconstituted in $100 \mu \mathrm{L}$ of methanol, vortex mixed for $2 \mathrm{~min}$, and centrifuged at $704 \times g$ for $10 \mathrm{~min}$ at $20^{\circ} \mathrm{C}$. The supernatant was transferred to a glass vial and a $5 \mu \mathrm{L}$ aliquot was injected into the UPLC-MS/MS system.

\section{Pharmacokinetics study}

RAGE (100 mg) was dissolved in ethanol solution (ethanol:saline $=1: 9, v / v$ ) and was orally administered to $\mathrm{SD}$ rats $(n=6)$. Blood samples $(-0.3 \mathrm{~mL})$ were collected from a jugular vein in heparinized tubes before the administration, and $0.17,0.5,1,2,4,6,8,12,24$, or $48 \mathrm{~h}$ after intragastric administration. Collected blood samples were immediately centrifuged at $704 \times g$ for $10 \mathrm{~min}$, and $150 \mu \mathrm{L}$ aliquots of supernatant plasma were transferred to clean tubes and stored at $-20{ }^{\circ} \mathrm{C}$ until analysis. The pharmacokinetic parameters of decursin were calculated using plasma concentration of decursin versus time data using PKSolver (a free add-in program for Microsoft Excel) [23].

\section{Statistical analysis}

Data are presented as the mean \pm standard error and analyzed using SigmaPlot 12.0 (Systat, CA, USA). Statistical differences between groups were evaluated using ANOVA followed by the Dunnett's test, and values of $p<0.05$ were considered significant.

\section{Results}

HPLC analysis of RAGE and its standard compounds

Four marker compounds were confirmed in RAGE by their retention times and UV spectra compared with those of the standard compounds on the HPLC chromatogram (Fig. 1) and their content in the extract was as follows: $11.303 \pm 0.188 \mathrm{mg} / \mathrm{g}$ for chlorogenic acid, $25.923 \pm 0.241 \mathrm{mg} / \mathrm{g}$ for nodakenin, $0.191 \pm 0.005 \mathrm{mg} / \mathrm{g}$ for imperatorin, and $141.130 \pm 1.309 \mathrm{mg} / \mathrm{g}$ for decursin (Table 1).

\section{Effects of RAGE on infarct volumes and NDS}

To study the effect of RAGE, mice were pre-treated with RAGE before induction of ischemic stroke by $90 \mathrm{~min}$ tMCAO. RAGE (1000 mg/kg bw) was administered once ( $1 \mathrm{~h}$ prior to $\mathrm{tMCAO}$ ) or twice ( $1 \mathrm{~h}$ prior to $\mathrm{tMCAO}$ and $24 \mathrm{~h}$ earlier). The more effective regimen was the double $1000 \mathrm{mg} /(\mathrm{kg} \mathrm{bw} \cdot \mathrm{d})$ dose (Fig. 2a, b). The total infarct volume percentile in the ipsilateral brain hemisphere in mice treated twice with RAGE at $1000 \mathrm{mg} /(\mathrm{kg} \mathrm{bw} \cdot \mathrm{d})$ was $23.78 \pm 1.14 \%$, and that of the untreated tMCAO group was $39.16 \pm 1.58 \%$ (Fig. $2 b$ ).

No significant reduction in the amount of brain edema was observed in the groups pre-treated with $1000 \mathrm{mg} /(\mathrm{kg} \mathrm{bw} \cdot \mathrm{d})$ RAGE once $\left(19.48 \pm 7.01 \mathrm{~mm}^{3}\right)$ or twice $\left(14.01 \pm 2.19 \mathrm{~mm}^{3}\right)$, when compared with the 
amount of edema seen in the untreated tMCAO group $\left(15.88 \pm 0.93 \mathrm{~mm}^{3}\right)$ (Fig. 2c). No significant changes were observed in the neurological deficit scores (NDS) and body weight changes between the tMCAO-induced groups (Fig. 2d, e, respectively).

\section{Effects of RAGE on protein expressions}

As pre-treatment of $1000 \mathrm{mg} / \mathrm{kg}$ bw RAGE twice was found to be more effective than the single treatment, we assessed the expression of cellular stress-related molecules in the ischemic ipsilateral cerebral cortex and hippocampus. tMCAO showed no significant changes of cellular stress-related protein expressions such as extracellular-signal-regulated kinase (ERK), c-Jun $\mathrm{N}$-terminal kinase (JNK), and p38 mitogen-activated protein kinase (p38) (Fig. 3a-c); but interestingly, pre-treatment with RAGE increased phosphorylation of JNK (Fig. 3b). Hippocampal protein phosphorylation of ERK was increased by tMCAO which was regulated by pre-treatment of $1000 \mathrm{mg} /(\mathrm{kg}$ bw.d) RAGE twice (Fig. 3d). ERK, JNK, and p38 mediate mitogen-activated protein kinase (MAPK) pathways, and MAPK is an intracellular signal-mediating molecule involved in various cell activities including cell proliferation, differentiation, survival, death, and deformation [24-28]. Thus, MAPK signaling was partially activated by ERK in the TMCAO group, and the activated signaling pathways were thought to be regulated by RAGE treatment (Fig. 3d).

\section{Effects of RAGE on histological changes during ischemic} brain tissue injury

To evaluate the neuroprotective effect of RAGE on ischemic neuronal damage, we investigated the morphological changes to neuronal cells in the ischemic ipsilateral hemisphere of tMCAO-induced mice. In the sham-operated group, cresyl violet staining, also known as Nissl staining, indicated that the neuronal cells were intact, with a morphologically well-arranged cytoplasm and nucleus; however, in the tMCAO group, the neuronal cells in the hippocampal CA1 region were apoptotic, showing aberrant morphology with sparsely positioned cells (white arrows, Fig. 4a). In the RAGE-treated group, the cells were similar to those of the sham-operated group (black arrows, Fig. 4a). Hematoxylin and eosin (H\&E) staining indicated that cell density of the hippocampal CA1 region in the $\mathrm{MCAO}$-induced group was slightly decreased, whereas in the group pre-treated with RAGE for two consecutive days cell density was similar to that of the sham-operated group (Fig. 4a). Hippocampal CA2 and CA3 regions showed a similar result as that of the CA1 region (Additional file 1: Figure S1).

\section{Effects of RAGE on pro-inflammatory cytokine levels}

Immunohistochemistry (IHC) staining in the ischemic ipsilateral hemisphere showed that TNF- $\alpha$ and interleukin 6 (IL-6) were highly expressed in the hippocampal CA1 region (red arrows indicate TNF- $\alpha$ and IL- 6 positive stains, Fig. 4a). The expression levels of TNF- $\alpha$ and

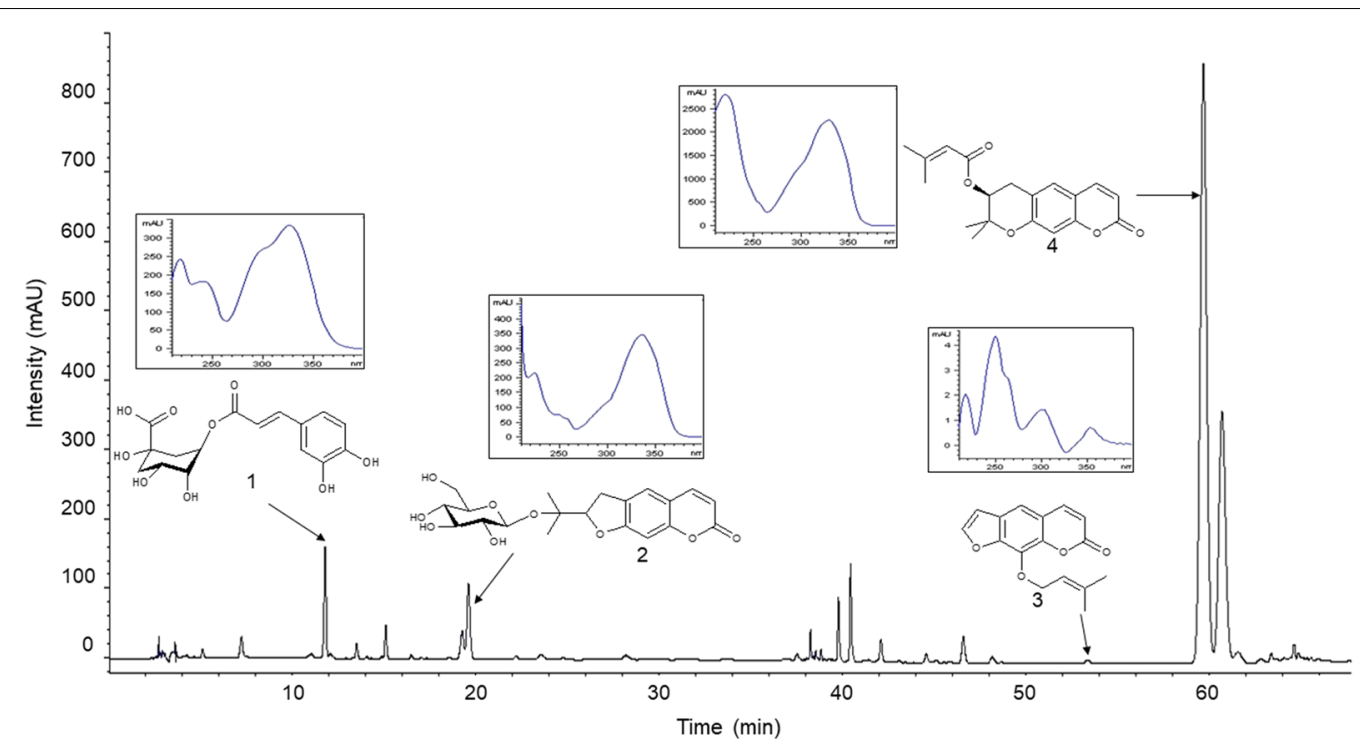

Fig. 1 Chromatograms of RAGE at the UV wavelength of $250 \mathrm{~nm}$ with chemical structures and UV spectra of the marker compounds: 1 . chlorogenic acid, 2. nodakenin, 3. imperatorin, and 4. decursin 
Table 1 Qualitative identification and quantitative calibration of four marker compounds in RAGE

\begin{tabular}{|c|c|c|c|c|c|}
\hline Compound & $\lambda_{\max }(\mathrm{nm})$ & $t_{R}(\min )$ & Calibration equation & $r^{2}$ & $\begin{array}{l}\text { Calibration } \\
\text { range }(\mu \mathrm{g} / \\
\mathrm{mL})\end{array}$ \\
\hline Chlorogenic acid (1) & 218,326 & 11.76 & $y=23.414 x-4.5529$ & 0.9991 & $0.78-50.00$ \\
\hline Nodakenin (2) & 224,336 & 19.58 & $y=23.670 x+8.2782$ & 1.0000 & $3.13-200.00$ \\
\hline Imperatorin (3) & $218,250,302,354$ & 53.33 & $y=52.339 x+0.5308$ & 0.9998 & $0.31-10.00$ \\
\hline Decursin (4) & 222,326 & 59.67 & $y=56.013 x+82.431$ & 1.0000 & $9.38-600.00$ \\
\hline
\end{tabular}
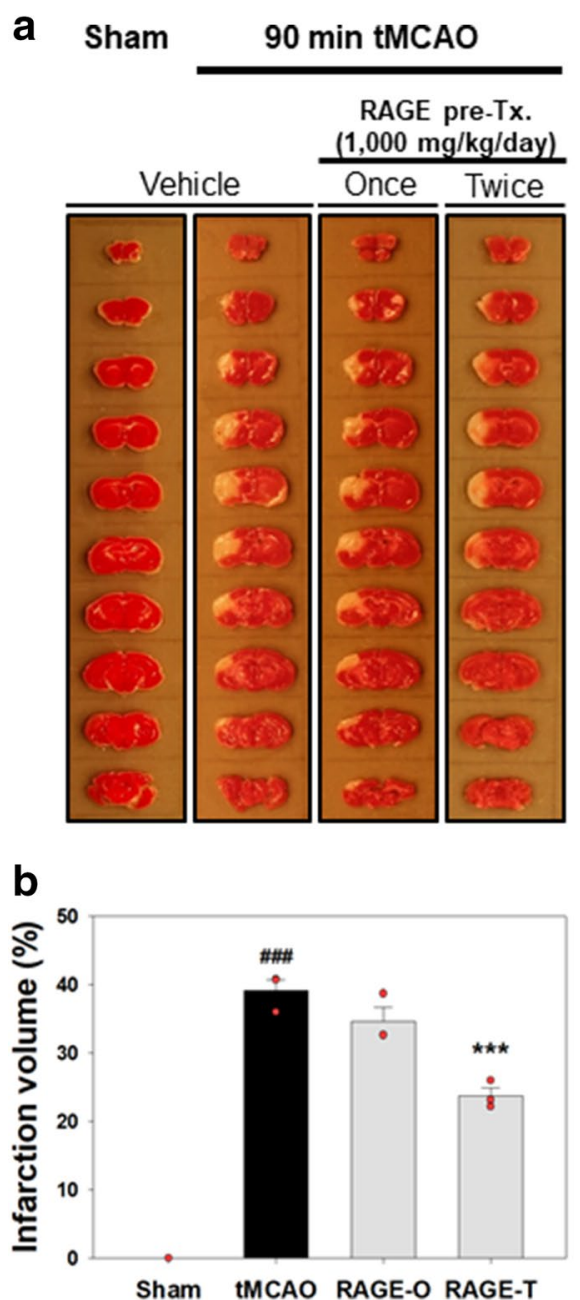

C

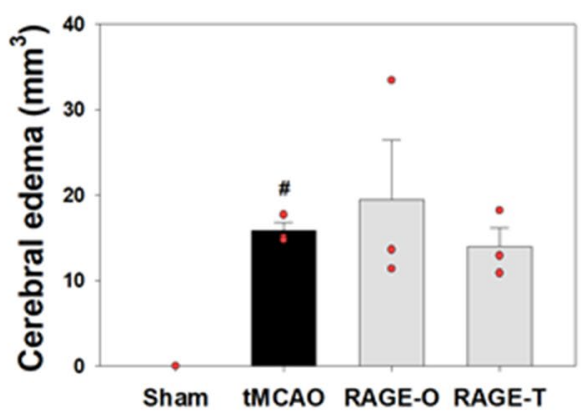

d

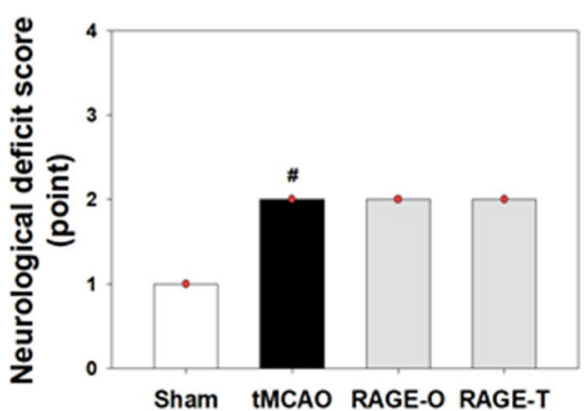

e

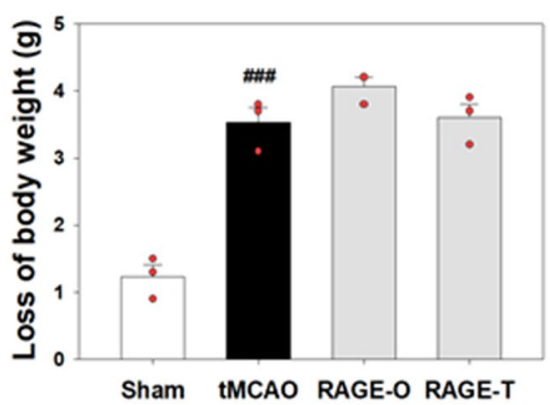

Fig. 2 Measurements of infarction volume, edema area, NDS, and body weight change. a representative photographs of TTC-stained brain slices $(1 \mathrm{~mm})$ showing the infarct area $24 \mathrm{~h}$ after $\mathrm{MMCAO}$ treatment; $\mathbf{b}$ quantitative analysis of the total infarct volume; c quantitative analysis of the total edema area; $\mathbf{d}$ NDS; e change in body weight. Once, single RAGE treatment; twice, RAGE treatment for two consecutive days at $1000 \mathrm{mg} /(\mathrm{kg}$ bw.d) RAGE. All data are expressed as mean \pm standard error $(n=3) . "{ }^{*} p<0.05$, \#\#\# $p<0.001$ vs. sham-operated group; ${ }^{* * *} p<0.001$ vs. tMCAO group

IL-6 were lower in the RAGE-treated group than in the tMCAO group.

The effects of pre-treatment with RAGE twice on hippocampal inflammation after tMCAO-mediated injury were evaluated by measuring the levels of pro-inflammatory cytokines, TNF- $\alpha$ and IL-6. The ischemic ipsilateral hippocampus hemisphere of mice with $\mathrm{tMCAO}$ exhibited higher concentrations of TNF- $\alpha$ $(675.87 \pm 20.86 \mathrm{pg} / \mathrm{mL})$ and IL-6 $(53.25 \pm 2.44 \mathrm{pg} /$ $\mathrm{mL}$ ) than that in the sham-operated mice (TNF- $\alpha$; 
$480.73 \pm 18.33 \mathrm{pg} / \mathrm{mL}, \mathrm{IL}-6 ; 32.35 \pm 1.44 \mathrm{pg} / \mathrm{mL})$. However, RAGE pre-treatment significantly suppressed the expression of TNF- $\alpha(512.86 \pm 32.96 \mathrm{pg} / \mathrm{mL})$ and IL-6 $(39.07 \pm 0.97 \mathrm{pg} / \mathrm{mL})$ (Fig. 4b, c). The serum levels of TNF- $\alpha$ and IL- 6 showed no change with tMCAOmediated brain injury (Additional file 1: Figure S2a, b); however, RAGE pre-treatment for two consecutive days significantly increased serum level of IL-6 (Additional file 1: Figure S2a, b).

\section{Pharmacokinetic change of decursin, the major compound of RAGE}

Decursin content in the plasma samples was determined after a single administration of RAGE to SD rats (100 mg/rat), a dose corresponding to that administered to tMCAO mice. The mean plasma concentration-time profile of decursin is shown in Fig. 5. The pharmacokinetic parameters, including area under the plasma concentration-time curve, maximum plasma concentration, time to reach the maximum plasma concentration, $t_{1 / 2}$, mean residence time, and last measured concentration are shown in Table 2.

\section{Discussion}

Stroke is the second most common cause of death in the world. The most common type of stroke is ischemic stroke, a major neurological disorder that causes physical and psychological disorders [1-5]. As there is little regeneration of brain tissue after ischemic injury, there is a high possibility of a stroke relapse owing to cerebrovascular disorder. Therefore, drug therapy to improve ongoing risk factors is recommended for patients with post-ischemic brain dysfunction [29-31].

According to the theoretical basis of Korean medicine, pathological wind contains internal factors caused by liver dysfunction, fire is a stressful condition that can cause stroke, sputum can cause hyperlipemia or cerebral thrombosis, and a lack of life can cause a lack of blood circulation in the body [13, 32]. RAG has been widely used in various cardiovascular diseases as it has been

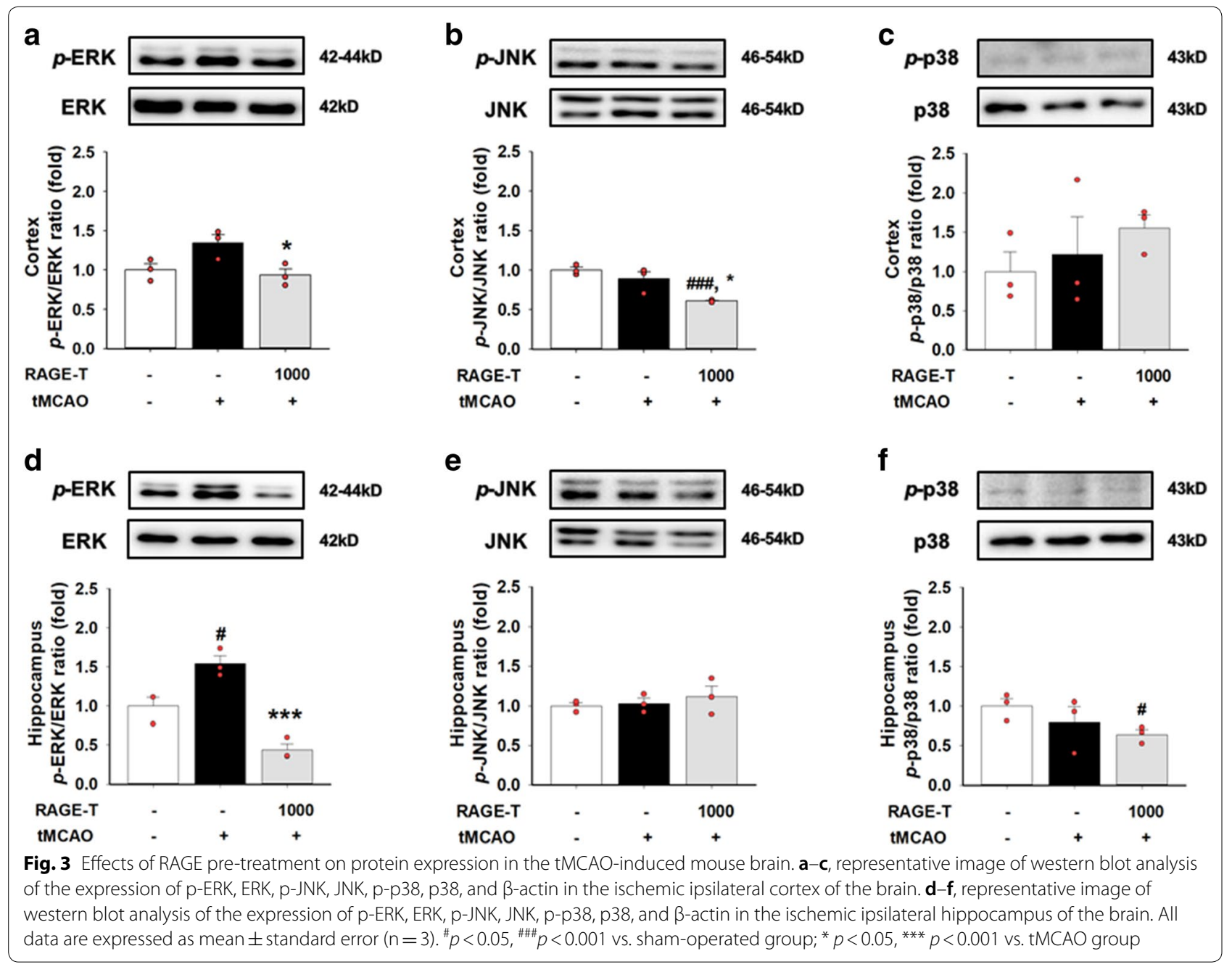



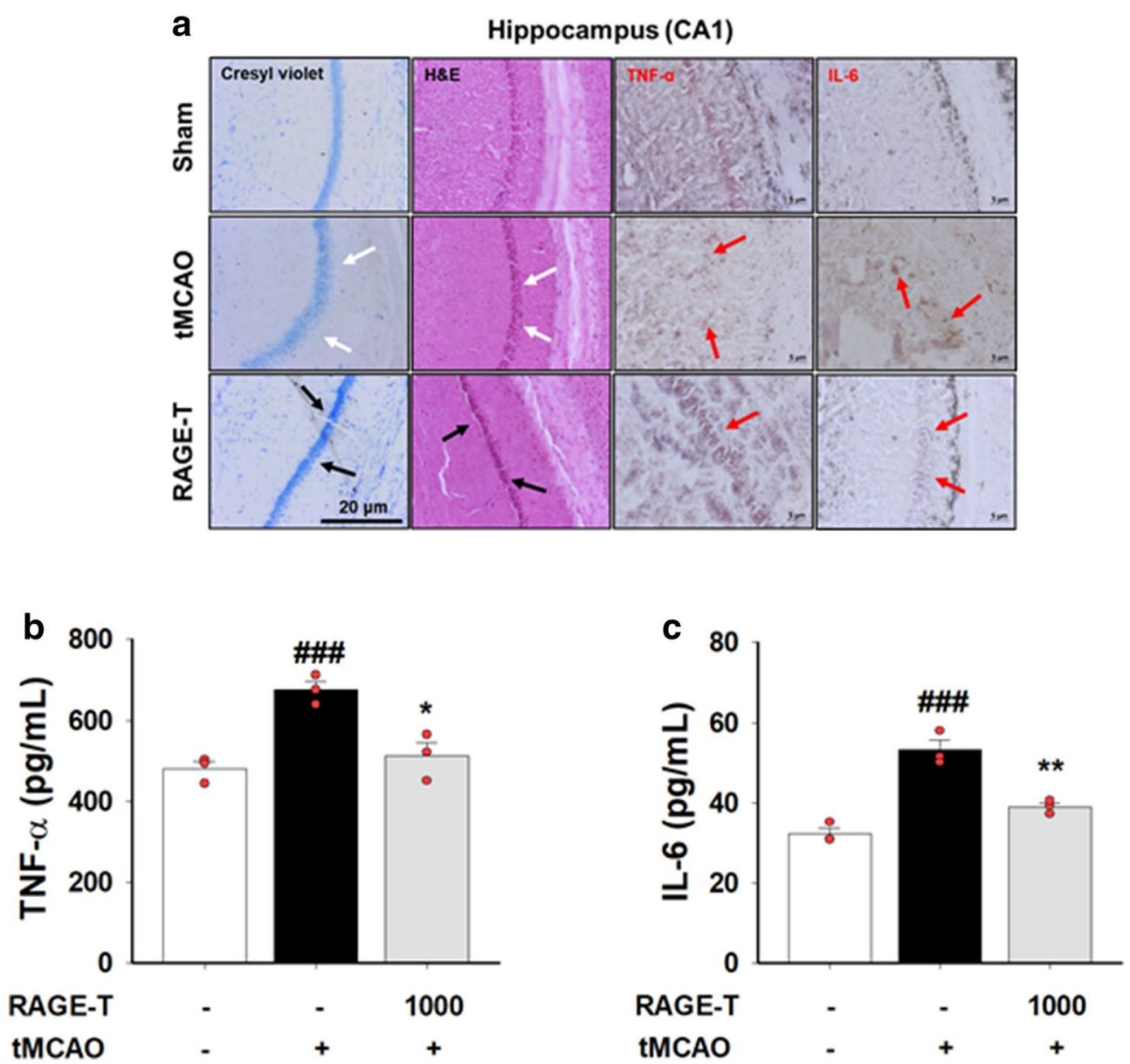

Fig. 4 Neuroprotective effects of RAGE on tMCAO-induced hippocampal CA1 cell death. A, representative photomicrographs of cresyl violet-stained, H\&E-stained, and IHC-stained (TNF- $a$ and IL-6, respectively) hippocampal CA1 region. B and C, quantitative analysis of the TNF-a and IL-6 expression in TMCAO-induced hippocampal CA1 region using the ELISA method. All data are expressed as mean $\pm s \operatorname{standard~error~}(n=3)$. \#\# $p<0.001$ vs. sham-operated group; ${ }^{*} p<0.05,{ }^{* *} p<0.01$ vs. tMCAO group

reported to affect cardiovascular retarded vasodilation, neuroglutamic acid toxicity, and memory impairment [13]. Therefore, these various neuroprotective properties indicate that RAG could be used to treat ischemic stroke.

There have been previous reports regarding RAG and its constituents' neuroprotective effects [13, 14, 25], and Shin et al. and Oh et al. reported that aqueous extracts of RAG attenuated cerebral damage and neuronal death in tMCAO-induced ischemic rats; however, they did not measured $\mathrm{rCBF}$ using laser Doppler flowmeter during the MCAO surgery [13,33]. However, to our knowledge, pharmacokinetics studies on effective dosage of RAG have not been conducted until now. Furthermore, Shin et al. used rats as experimental animal, applied single treatment using intraperitoneal injection [13]. In our preliminary study, however, we used mice applying experimental methods of Shin et al., but we failed to confirm the effect of RAGE in that experimental condition.
Therefore, we have newly identified conditions for the effect of RAGE in this study applying methanol as extraction solvent, and oral administration pathways. Thus, we demonstrated the pharmacological effects of RAGE in a previously reported modified ischemic stroke mouse model [22], and conducted a pharmacokinetic study with decursin, a major compound in the root of $A$. gigas.

RAGE reduced the total infarct volume induced by ischemic stroke when it was administered for two consecutive days prior to the ischemic stroke (Fig. $2 \mathrm{~A}$ and $2 \mathrm{~B})$, and the $t_{1 / 2}$ of decursin, a major bioactive compound of $A$. gigas, was $8.28 \mathrm{~h}$ after oral administration (Table 2), which suggests that the interval of administration should be strictly maintained to obtain high efficacy without adverse effects from RAGE. Although pre-treatment with RAGE once or twice showed no significant change in brain edema and NDS when compared to that in $\mathrm{TMCAO}$ group, administration of the treatment twice was 
considered to have higher efficacy than a single administration of the treatment. The dose of $1000 \mathrm{mg} /(\mathrm{kg} \mathrm{bw} \cdot \mathrm{d})$ of RAGE was tittered in our preliminary studies. A lower dose (under $300 \mathrm{mg} /(\mathrm{kg} \mathrm{bw} \cdot \mathrm{d})$ ) showed no effects and a higher dose (over 3,000 $\mathrm{mg} /(\mathrm{kg} \mathrm{bw} \cdot \mathrm{d})$ ) showed adverse effects; therefore, we assumed that $1000 \mathrm{mg} /(\mathrm{kg} \mathrm{bw} \cdot \mathrm{d})$ would be more appropriate for this study. A RAGE dose of $300 \mathrm{mg} /(\mathrm{kg} \mathrm{bw} \cdot \mathrm{d})$ is closer to the clinically applied and recommended dose for daily use; however, in this rodent study, we assessed one or two administrations of $1000 \mathrm{mg} /(\mathrm{kg}$ bw.d) RAGE.

The effect of RAGE pre-treatment on tMCAO-induced cerebral damage was also assessed histologically in the hippocampal region. The cresyl violet stain for neurons and the H\&E stain for nuclei and cytoplasm in the hippocampus CA1 region showed that pre-treatment with RAGE twice resulted in improved cell density and morphology when compared with that seen after treatment with tMCAO alone (Fig. 3a), and tMCAO-induced cerebral inflammation was inhibited by RAGE pre-treatment (Fig. 3sa-c). The cerebral inflammatory response induced by tMCAO did not seem to acutely affect parts of the body other than the brain (Additional file 1: Figure S2).

Corresponding results were obtained with western blotting in the ischemic ipsilateral hippocampus hemisphere showing inhibition of increased phosphorylation of ERK induced by tMCAO (Fig. 3d). MAPK is an intracellular signaling molecule with effects on cell migration, proliferation, and differentiation, and its relevant sub-molecules are ERK, JNK, and p38 [3436], and activation of ERK promotes cellular growth,
Table 2 Pharmacokinetic parameters of decursin after oral administration of RAGE

\begin{tabular}{lc}
\hline Parameter & Mean \pm SD \\
\hline $\mathrm{AUC}_{0-\infty}(\mathrm{ng} \cdot \mathrm{hr} / \mathrm{mL})$ & $1218.319 \pm 326.042$ \\
$\mathrm{AUC}_{0-t}(\mathrm{ng} \cdot \mathrm{hr} / \mathrm{mL})$ & $1186.935 \pm 323.500$ \\
$\mathrm{C}_{\text {max }}(\mathrm{ng} / \mathrm{mL})$ & $407.888 \pm 85.732$ \\
$T_{\max }(\mathrm{hr})$ & $1.750 \pm 1.255$ \\
$t_{1 / 2}(\mathrm{hr})$ & $8.283 \pm 0.538$ \\
$\mathrm{MRT}_{0-\infty}(\mathrm{hr})$ & $6.897 \pm 0.954$ \\
$\mathrm{MRT}_{0-t}(\mathrm{hr})$ & $5.433 \pm 0.581$ \\
$\mathrm{C}_{\text {last }}(\mathrm{ng} / \mathrm{mL})$ & $2.621 \pm 0.665$ \\
\hline
\end{tabular}

$\mathrm{AUC}_{0-t,}$ the area under the curve from 0 time to $48 \mathrm{~h} ; \mathrm{AUC}_{0-\infty}$, the area under the curve from 0 time to infinity; $C_{\max }$, the maximum drug concentration in plasma; $t_{1 / 2}$, half-life; $T_{\text {max }}$, time taken to reach maximum drug concentration in plasma; $\mathrm{MRT}_{0-\infty}(\mathrm{hr})$, mean residence time from 0 to infinity last measured concentration; $\mathrm{MRT}_{0-t}(\mathrm{hr})$, mean residence time from 0 time to $48 \mathrm{~h} ; \mathrm{C}_{\text {last }}$ last measured concentration

differentiation, or mitosis [26-28]. Ischemic ipsilateral hippocampal ERK signaling was significantly activated in the tMCAO group which was inhibited by RAGE pre-treatment (Fig. 3d). Interestingly, JNK signaling which activates apoptotic pathways by up-regulating pro-apoptotic genes [25-27] was down-regulated by RAGE pre-treatment (Fig. 3b).

Among the subgroups of the MAPK family, ERK is regarded as being related to cell survival; however, emerging evidence suggests the activation of ERK may lead to neuronal cell death, and inhibition of the ERK pathway is considered neuroprotective against

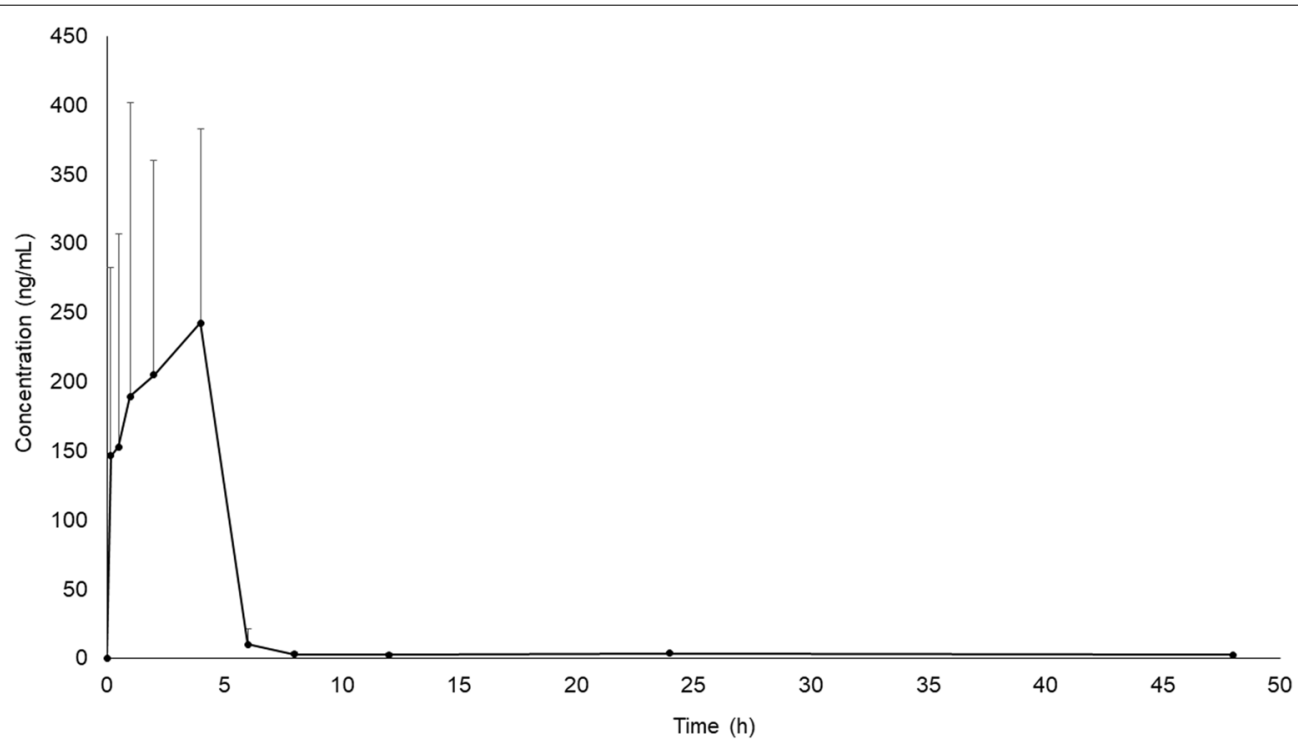

Fig. 5 Chemical structures of decursin and schizandrin (IS), and mean plasma concentration-time curves of decursin after the oral administration of RAGE. Results are presented as mean \pm standard $(n=6)$ 
oxidative stress [37]. Till date, the role of ERK remains controversial [34-37]. In our study, $p$-ERK was overexpressed in the MCAO-induced ipsilateral brain, and was down-regulated with the administration of RAGE (Fig. 3d). Although it is not yet possible to draw a definite conclusion, it may be assumed that ERK, a cell survival related signal, is activated by cell death related signals induced by $\mathrm{MCAO}$, and was down-regulated by reduced brain injury owing to the administration of RAGE. Son et al. reported that activities of ERK and p38 in colon carcinoma cells were inhibited by decursin and decursinol which are the main components of $A$. gigas [38], thus supporting the role of RAGE in ERKrelated molecular mechanism.

Plasma concentration of a bioactive compound after oral administration in vivo can provide important information in deciding the bioactive dose of herbal extract for rodents of a brain disease model. Decursin was found to be the most abundant in RAGE in our experiment, as previously reported [39], and thus, plasma concentrations of decursin were determined after oral administration of RAGE to SD rats. Although mice were used in the tMCAO experiment, we chose rats to quantify the plasma concentrations of decursin, as rats are more advantageous than mice for continuous collection of blood over a period of $24 \mathrm{~h}$. The dose administered to tMCAO-induced mice was converted to $100 \mathrm{mg} / \mathrm{rat}$ using calculations described in Nair et al. [40]. Pharmacokinetic variables, such as area under the plasma concentration-time curve, time to reach the maximum plasma concentration, maximum plasma concentration, $t_{1 / 2}$, and mean residence time, were calculated as physiological parameters after oral administration of RAGE to the non-diseased rat model. However, no studies have previously reported the pharmacokinetics of the compounds of A. gigas at their bioactive concentrations in animal models with a specific disease. Therefore, further studies on the pharmacokinetic changes in bioactive compounds of RAGE in diseased animal models are necessary for decisions on effective dosage.

\section{Conclusions}

Mice administered RAGE at $1000 \mathrm{mg} /(\mathrm{kg} \mathrm{bw} \cdot \mathrm{d})$ for two consecutive days before the tMCAO operation had less neuronal cell death than those that were not administered RAGE prior to the operation. This protective effect involved MAPK-related cellular stress signals. Decursin was the most abundant compound in RAGE and its pharmacokinetic parameters were determined physiologically. This study provides preclinical evidence for the neuroprotective use of $A$. gigas in the treatment of ischemic stroke.

\section{Supplementary information}

Supplementary information accompanies this paper at https://doi. org/10.1186/s13020-020-00383-1.

Additional file 1. Details regarding cell density in the hippocampal region and serum level changes in TNF- $a$ and IL- 6 with $\mathrm{tMCAO-mediated}$ brain injury and pre-treatment with RAGE are available in additional information.

\section{Abbreviations}

ANOVA: Analysis of variance; ERK: Extracellular-signal-regulated kinase; H\&E: Hematoxylin and eosin; IgG: Immunoglobulin G; IHC: Immunohistochemistry; IL-6: Interleukin 6; JNK: C-Jun n-terminal kinase; MAPK: Mitogen-activated protein kinase; MCA: Middle cerebral artery; NDS: Neurological deficit scores; p38: P38 mitogen-activated protein kinase; RAG: Root of Angelica gigas; RAGE: Methanolic extract of the root of A. gigas; rCBF: Relative cerebral blood flow; $t_{1 / 2}$ : Half-life time; tMCAO: Transient middle cerebral artery occlusion; TTC: 2,3,5-Triphenyl-tetrazolium chloride.

\section{Acknowledgment \\ Not applicable.}

\section{Authors' contributions}

Conceptualization: JHK and SC. Methodology: SEL and SC. Investigation: SEL, JHK, and SC. Data analysis: SEL and CL. Writing-Original Draft: SEL. WritingReview \& Editing: SEL, JHK, CL, and SC. Supervision: SC. All authors read and approved the final manuscript.

\section{Funding}

Not applicable.

Availability of data and materials

Please contact corresponding authors for data requests.

\section{Ethics approval and consent to participate}

All procedures in this study were approved and supervised by Ethics Committee for Animal Care and Use at Pusan National University (Approval No. PNU-2018-2113 and PNU-2019-2124), certified by the Korean Association of Laboratory Animal Care.

\section{Consent for publication}

Not applicable.

\section{Competing interest}

The authors declare no conflict of interest.

\section{Author details}

1 Department of Korean Medicine, School of Korean Medicine, Yangsan Campus of Pusan National University, Yangsan 50612, Republic of Korea. ${ }^{2}$ Department of Medicine, College of Medicine, Dongguk University, Goyang 10326, Republic of Korea.

Received: 29 June 2020 Accepted: 18 September 2020

Published online: 22 September 2020

References

1. Ingall T. Stroke-incidence, mortality, morbidity and risk. J Insur Med. 2004;36:143-52.

2. Malik S, Wong ND, Franklin SS, Kamath TV, L'Italien GJ, Pio JR, Williams GR. Impact of the metabolic syndrome on mortality from coronary heart disease, cardiovascular disease, and all causes in United States adults. Circulation. 2004;1 10:1245-50.

3. Mittal SH, Goel D. Mortality in ischemic stroke score: A predictive score of mortality for acute ischemic stroke. Brain Circ. 2017;3:29-34. 
4. Shah B, Bartaula B, Adhikari J, Neupane HS, Shah BP, Poudel G. Predictors of in-hospital mortality of acute ischemic stroke in adult population. $J$ Neurosci Rural Pract. 2017:8:591-4

5. Ramirez L, Kim-Tenser MA, Sanossian N, Cen S, Wen G, He S, Mack WJ, Towfighi A. Trends in acute ischemic stroke hospitalizations in the United States. J Am Heart Assoc. 2016. https://doi.org/10.1161/JAHA.116.003233.

6. Huang J, Upadhyay UM, Tamargo RJ. Inflammation in stroke and focal cerebral ischemia. Surg Neurol. 2006;66:232-45.

7. Clark RK, Lee EV, White RF, Jonak ZL, Feuerstein GZ, Barone FC. Reperfusion following focal stroke hastens inflammation and resolution of ischemic injured tissue. Brain Res Bull. 1994;35:387-92.

8. Sims NR, Muyderman H. Mitochondria, oxidative metabolism and cell death in stroke. Biochim Biophys Acta. 2010;1802:80-91.

9. Hachinski V, Azarpazhooh MR. Stroke is a burdensome but preventable brain disorder. Lancet Neurol. 2016;15:892-3.

10. Donnan GA, Fisher M, Macleod M, Davis SM. Stroke. The Lancet. 2008;371:1612-23.

11. Hilbrich L, Truelsen T, Yusuf S. Stroke and cardiovascular diseases: the need for a global approach for prevention and drug development. Int J Stroke. 2007:2:104-8.

12. Lenfant C. Can we prevent cardiovascular diseases in low- and middleincome countries? Bull World Health Organ. 2001;79:980-3.

13. Shin YJ, Park YK. Effects of different parts of Angelica gigas Nakai on brain damages and neuronal death in transient middle artery occlusion/ reperfusion-induced ischemic rats. Kor J Herbol. 2014;29:85-93.

14. Kang SY, Kim YC. Neuroprotective coumarins from the root of Angelica gigas: structure-activity relationships. Arch Pharm Res. 2007;30:1368-73.

15. Sarker SD, Nahar L. Natural medicine: the genus Angelica. Curr Med Chem. 2004:11:1479-500.

16. Joo SS, Park D, Shin S, Jeon JH, Kim TK, Choi YJ, Lee SH, Kim JS, Park SK, Hwang BY, Lee DI, Kim Y. Anti-allergic effects and mechanisms of action of the ethanolic extract of Angelica gigas in dinitrofluorobenzene-induced inflammation models. Environ Toxicol Pharmacol. 2010;30:127-33.

17. Lee S, Lee YS, Jung SH, Shin KH, Kim BK, Kang SS. Anti-tumor activities of decursinol angelate and decursin from Angelica gigas. Arch Pharm Res. 2003;26:727-30

18. Park SJ, Jung JM, Lee HE, Lee YW, Kim DH, Kim JM, Hong JG, Lee CH, Jung $I H$, Cho Y, Jang DS, Ryu JH. The memory ameliorating effects of INM-176, an ethanolic extract of Angelica gigas, against scopolamine- or A 1 1-42-induced cognitive dysfunction in mice. J Ethnopharmacol. 2012;143:611-20.

19. Seong G, Baek M, Lee Y, Won J. Quality evaluation of Angelica gigas Nakai with different drying methods and different root parts. Kor J Herbol. 2018:33:85-91.

20. Li L, Yang Y, Zheng J, Cai G, Lee Y, Du J. Decursin attenuates the amyloid-beta-induced inflammatory response in PC12 cells via MAPK and nuclear factor-kappaB pathway. Phytother Res. 2018:32:251-8

21. Chang SN, Khan I, Dey DK, Cho KH, Hwang BS, Bae KB, Kang SC, Park JG. Decursinol angelate ameliorates 12-O-tetradecanoyl phorbol-13-acetate (TPA)-induced NF-kappaB activation on mice ears by inhibiting exaggerated inflammatory cell infiltration, oxidative stress and pro-inflammatory cytokine production. Food Chem Toxicol. 2019;132:110699.

22. Lee SE, Lim C, Lee M, Kim CH, Kim H, Lee B, Cho S. Assessing neuroprotective effects of Glycyrrhizae Radix et Rhizoma extract using a transient middle cerebral artery occlusion mouse model. J Vis Exp. 2018. https:// doi.org/10.3791/58454.

23. Zhang Y, Huo M, Zhou J, Xie S. PKSolver: An add-in program for pharmacokinetic and pharmacodynamic data analysis in Microsoft Excel. Comput Methods Programs Biomed. 2010;99:306-14.

24. Guo C, Yang M, Jing L, Wang J, Yu Y, Li Y, Duan J, Zhou X, Li Y, Sun Z. Amorphous silica nanoparticles trigger vascular endothelial cell injury through apoptosis and autophagy via reactive oxygen species-mediated MAPK BCl-2 and PI3K/Akt/mTOR signaling. Int J Nanomed. 2016;11:5257-76.

25. Li L, Du JK, Zou LY, Wu T, Lee YW, Kim YH. Decursin isolated from Angelica gigas Nakai rescues PC12 cells from amyloid beta-protein-induced neurotoxicity through Nrf2-mediated upregulation of heme oxygenase-1: Potential roles of MAPK. Evid Based Complement Alternat Med. 2013;2013:467245.

26. Chuang SM, Wang IC, Yang JL. Roles of JNK, p38 and ERK mitogen-activated protein kinases in the growth inhibition and apoptosis induced by cadmium. Carcinogenesis. 2000;21:1423-32.
27. Chuang SM, Liou GY, Yang JL. Activation of JNK, p38 and ERK mitogenactivated protein kinases by chromium (VI) is mediated through oxidative stress but does not affect cytotoxicity. Carcinogenesis. 2000;21:1491-500.

28. Plotnikov A, Zehorai E, Procaccia S, Seger R. The MAPK cascades: signaling components, nuclear roles and mechanisms of nuclear translocation. Biochim Biophys Acta. 2011;1813:1619-33.

29. Gu Z, Kaul M, Yan B, Kridel SJ, Cui J, Strongin A, Smith JW, Liddington RC, Lipton SA. S-nitrosylation of matrix metalloproteinases: signaling pathway to neuronal cell death. Science. 2002;297:1186-90.

30. Sattler R, Tymianski M. Molecular mechanisms of glutamate receptormediated excitotoxic neuronal cell death. Mol Neurobiol. 2001;24:107-29.

31. Dunn KM, Renic M, Flasch AK, Harder DR, Falck J, Roman RJ. Elevated production of 20-HETE in the cerebral vasculature contributes to severity of ischemic stroke and oxidative stress in spontaneously hypertensive rats. Am J Physiol Heart Circ Physiol. 2008;295:H2455-H24652465.

32. Cheng SC, Lin CH, Chang YJ, Lee TH, Ryu SJ, Chen CH, Hung YC. Fire-heat and Qi deficiency syndromes as predictors of short-term prognosis of acute ischemic stroke. J Altern Complement Med. 2013;19:721-8.

33. Oh TW, Park KH, Jung HW, Park YK. Neuroprotective effect of the hairy root extract of Angelica gigas Nakai on transient focal cerebral ischemia in rats through the regulation of angiogenesis. BMC Complement Altern Med. 2015;15:101.

34. Gao X, Zhang H, Takahashi T, Hsieh J, Liao J, Steinberg GK, Zhao H. The Akt signaling pathway contributes to postconditioning's protection against stroke; the protection is associated with the MAPK and PKC pathways. J Neurochem. 2008;105:943-55.

35. Sawe N, Steinberg G, Zhao H. Dual roles of the MAPK/ERK1/2 cell signaling pathway after stroke. J Neurosci Res. 2008;86:1659-69.

36. Sun J, Nan G. The mitogen-activated protein kinase (MAPK) signaling pathway as a discovery target in stroke. J Mol Neurosci. 2016;59:90-8.

37. Lu K, Cho CL, Liang CL, Chen DS, Liliang PC, Wang SY, Chen HJ. Inhibition of the MEKJERK pathway reduces microglial activation and interleukin1-beta expression in spinal cord ischemia/reperfusion injury in rats. J Thorac Cardiovasc Surg. 2007;133:934-41.

38. Son SH, Park KK, Park SK, Kim YC, Kim YS, Lee SK, Chung WY. Decursin and decursinol from Angelica gigas inhibit the lung metastasis of murine colon carcinoma. Phytother Res. 2011;25:959-64.

39. Jeong SY, Kim HM, Lee KH, Kim KY, Huang DS, Kim JH, Seong RS. Quantitative analysis of marker compounds in Angelica gigas, Angelica sinensis, and Angelica acutiloba by HPLC/DAD. Chem Pharm Bull. 2015;63:504-11.

40. Nair A, Jacob S. A simple practice guide for dose conversion between animals and human. J Basic Clin Pharm. 2016;7:27-31.

\section{Publisher's Note}

Springer Nature remains neutral with regard to jurisdictional claims in published maps and institutional affiliations.

Ready to submit your research? Choose BMC and benefit from:

- fast, convenient online submission

- thorough peer review by experienced researchers in your field

- rapid publication on acceptance

- support for research data, including large and complex data types

- gold Open Access which fosters wider collaboration and increased citations

- maximum visibility for your research: over 100M website views per year

At BMC, research is always in progress.

Learn more biomedcentral.com/submissions 